\title{
HELIOS Carrier: Tail-like Mechanism and Control Algorithm for Stable Motion in Unknown Environments
}

\author{
M. Guarnieri, P. Debenest, T. Inoh, K. Takita, H. Masuda, R. Kurazume, E. Fukushima and S. Hirose
}

\begin{abstract}
Mobile platforms when negotiating steps and stairs should be able to control theirs posture in order to avoid sudden tilting or falls. In particular, when considering applications for search and rescue operations where users have a very limited time of operation, the motion on stairs should be automated as much as possible. In this way operators can concentrate on their tasks (i.e. search of survivors and/or exploration of dangerous environments) rather than having to focus on the stability of the vehicle. A simple but very effective mechanism called "tail" is introduced. The mechanical design and its control method is presented together with several tests and experiments carried out with a simple tracked vehicle in real environments.
\end{abstract}

\section{INTRODUCTION}

Robotic platforms for Urban Search and Rescue Operations (USAR) must easily adapt to unstructured environments that consist of stairs, random steps and rubble. For this kind of applications the control of the CoG of the vehicle allows to negotiate several type of obstacles. To improve the vehicle terrain adaptability different mechanical solutions, together with the use of several sensors, have been so far proposed. Auxiliary tracked flippers are often utilized as in [11],[5], [10]. In other type of robotic platforms also an extra linkage with one active dof has been also utilized as in [1] and [2]. Also reconfigurable track profiles are adopted for obstacle negotiation [9],[8],[4],[3]. In many cases these solutions are characterized by complex hardware designs such as the use of extra treads and/or wheels together with sensors for their control. This might lead also to extra power consumption and to complex system architectures for the control of all the degrees of freedom. Thus if the dimensions of the platform are not increased, there is a limited space for the housing of sensor devices to be utilized for search and rescue operations.

In [6] the authors presented HELIOS VIII tracked vehicle equipped with a manipulator. The arm was proved to be very effective to assist the platform motion (i.e. control of the vehicle stability) when utilized as an extra linkage. The manipulator is utilized also for the connection of the vehicle and of an auxiliary simple tracked carrier to obtain articulated mobile configurations with high terrain adaptability. However, when operating in real scenarios, it is not always possible to make use of a unit equipped with a manipulator.

Guarnieri, Hirose, Debenest are with Faculty of Mechanical and Aerospace Engineering, Tokyo Institute of Technology, Tokyo, Japan guarnieri@robotics.mes.titech.ac.jp

Inoh and Takita are with HiBot Corporation, Kamata 2-10-1, Ohta, Tokyo 144-0052, JAPAN, Japan takita@hibot. co.jp

Masuda and Kurazume are with the Graduate School of Information Science and Electrical Engineering, Kyushu University, Tokyo, Japan
For this reason carriers must be capable up to a certain degree to move separately over stairs or steps.

In this research a simple and compact tail like mechanism, consisting of a flexible rubber pipe attached at the rear side of a basic tracked platform, is proposed. The tail permits the automatic motion support of the vehicle on steps and stairs by utilizing it to counteract the vehicle eventual tilting behavior. Nevertheless because of the particular mechanical design, plenty of space on the top of the platform is left for auxiliary batteries and array of sensors. Figure 1 shows one of the three carrier vehicles utilized for USAR missions. The addition of a tail improves significantly the mobility of crawler robots, while adding very little mass to the total mass of the robot. Compared to a normal extra rigid linkage with one dof, the use of flexible material for the tail permits to absorb eventual impacts without damaging the structure. In addition, a flexible tail would be less likely to become stuck among obstacles when the robot is moving in unstructured environments.

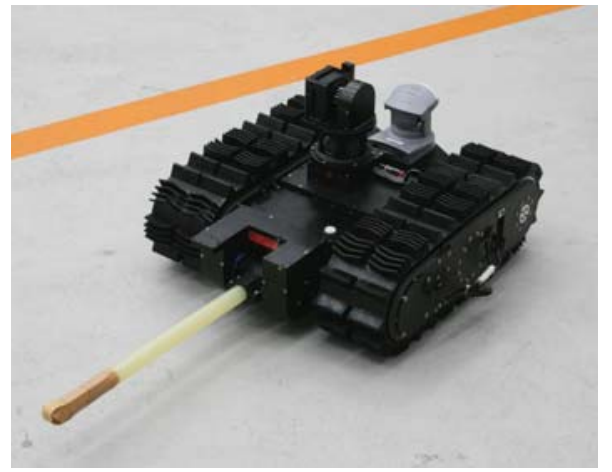

Fig. 1. Tracked platform equipped with tail like mechanism

The tail system consists of one dof. However, as explained in the experiments section, also the passive tail type with no actuation was proved to be very effective.

The paper is organized as follows: in the next section a basic geometric analysis of the proposed mechanism is introduced. Afterward the mechanical design and the control algorithm are explained in details. The effectiveness of the tail like solution is then validated throughout several experiments on prototypes and in real urban areas.

\section{Problem Analysis}

The following analysis has been carried out considering as a model for the tail a rigid fixed linkage. Further analysis considering the type of material and therefore the tail 
flexibility can be carried out. However this first basic study serves to give an idea of the dimensions and position of the extra linkage for stairs climbing.

For a crawler robot to climb stairs in a stable way, the projection of its center of mass must be always ahead of the pivoting point. The pivoting point is the edge of the stair around which the robot would rotate if its center of mass were located in the extreme back of the crawlers. While climbing stairs, the pivoting point corresponds to the lowest edge the crawlers are touching. Figure 2 shows the pivot point $\mathrm{P}$ at the most critical moment of stair climbing, when the projection of the center of gravity $(\mathrm{CG})$ of the robot is exactly over the pivot point. If the projection of the CG falls slightly to the left, the robot will lose balance and tilt backwards. However, if the projection of the CG is on the right of the pivot point, the robot will be in a statically stable posture.
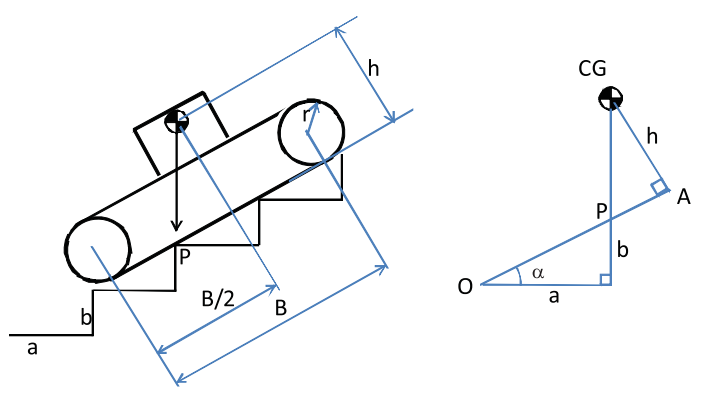

Fig. 2. Boundary condition for stable climbing of stairs

With considering Figure 2, for the projection of the center of mass to be always ahead of the pivoting point:

$$
\frac{B}{2} \cos (\alpha)-h \sin (\alpha) \geq a
$$

Thus, the two following relations are obtained:

$$
\begin{aligned}
& B>2 \sqrt{a^{2}+b^{2}}+\frac{2 b h}{a} \\
& h<\frac{a}{b}\left(\frac{B}{2}-\sqrt{a^{2}+b^{2}}\right)
\end{aligned}
$$

Making use of equations 2 and 3, the minimum necessary length of the crawler body for given heights of the center of mass to perform stable climbing of the stairs can be obtained.

\section{A. Tracked Robot equipped with TAIL mechanism}

If the crawler robot is equipped with a tail-like structure, it may be able to climb stairs and other obstacles that were previously inaccessible. The tail would function as an extension of the crawlers, but without adding much mass to the robot. In addition, if the tail can be rotated around its base in the pitch direction, it may help the crawler to achieve stable climbing on stairs that were previously impossible to climb.

To estimate the necessary length of the tail mechanism, consider the variables depicted in figure 3 . The crawler will become able to climb the steps without the support of the tail once the projection of its center of gravity reaches the pivot point $\mathrm{P}$. Until that moment, an additional support is necessary. This support is provided by the tail mechanism, which extends until the edge of the previous step.

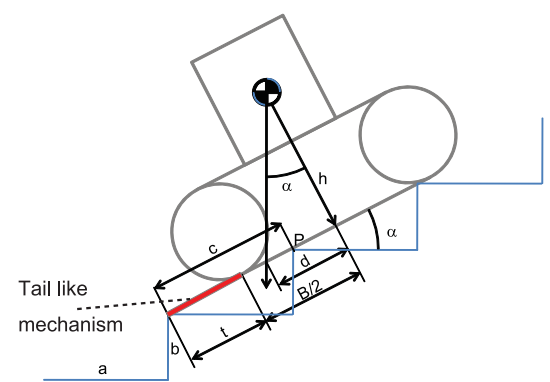

Fig. 3. Stair-climbing supported by tail mechanism

The distance between the center of the crawler and the projection of the center of gravity measured along the base of the crawler, $\mathrm{d}$, is given by the following equation:

$$
\tan (\alpha)=\frac{b}{a}=\frac{d}{h}
$$

Thus

$$
d=\frac{b h}{a}
$$

Since

$$
c=t+\left(\frac{B}{2}-d\right)
$$

the length of the tail $t$ is given by:

$$
t=c+\left(d-\frac{B}{2}\right)
$$

Even if the projection of the center of gravity falls behind the edge of the lower edge, as shown in figure 4, the same equation is valid, resulting in a longer tail that touches the edge of the previous step (i.e. previous to the projection of the center of gravity).

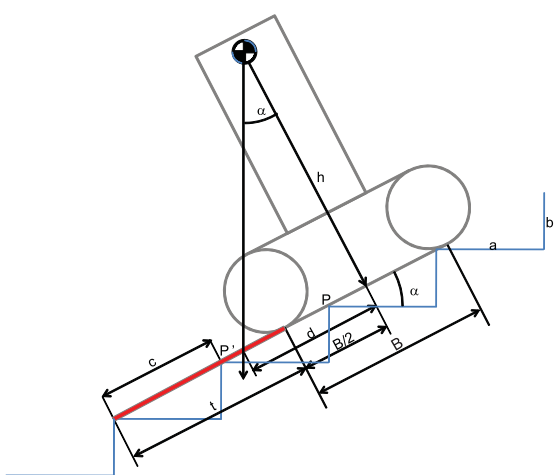

Fig. 4. Extend tail supports the climbing crawler even with high CG

According to figure 4 , if $d<\frac{B}{2}-c$, the crawler is always stable while climbing the stairs, and no tail is required. 
Table I shows possible configurations of crawler (with length $B$ and height of center of gravity $h$ ) and tail mechanism (of length $\mathrm{t}$ ) for stable climbing of standard stairs.

TABLE I

NECESSARY LENGTH AND ANGLE OF TAIL FOR PERFORMING STABLE CLIMBING

\begin{tabular}{|c|c|c|}
\hline $\mathrm{B}(\mathrm{mm})$ & $\mathrm{h}(\mathrm{mm})$ & $\mathrm{t}(\mathrm{mm})$ \\
\hline 395 & 87.5 & 171.6 \\
\hline 395 & 175 & 222.5 \\
\hline 395 & 262.5 & 273.4 \\
\hline 395 & 350 & 324.3 \\
\hline 395 & 437.5 & 375.2 \\
\hline
\end{tabular}

Note that all the crawler configurations presented in table I were not able to perform stable stair-climbing, as shown in the previous section. However, with the addition of a controlled tail, the robots become able to overcome the steps.

\section{B. Theoretical Comparison between standard and tail- equipped crawler robots}

Given the equations of the previous section, figure 5 shows the relation between the height of the center of gravity of the robot, h, and the necessary length of the crawler, B, with different lengths of tail, t. The physical meaning of these parameters is displayed in figure 4.

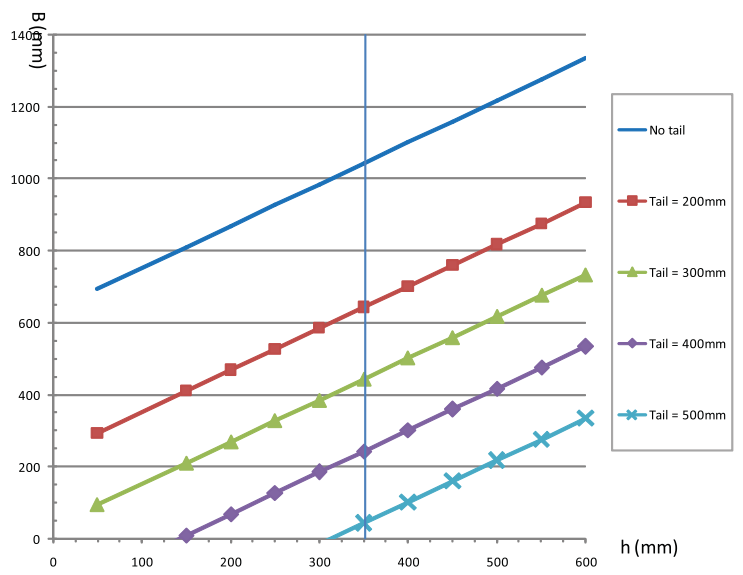

Fig. 5. Necessary length of crawler (B) against height of center of gravity of the robot $(\mathrm{h})$ for different lengths of tail mechanism (t)

Note that if no tail is employed, a robot with center of gravity $350 \mathrm{~mm}$ above the floor needs a crawler of at least $1044 \mathrm{~mm}$ of length to be able to climb steps. However, if a tail of $325 \mathrm{~mm}$ of length is added, the necessary length of the crawler is reduced to $395 \mathrm{~mm}$, without affecting its mobility when climbing stairs.

Since the mass of the crawlers per unit of length is much larger than that of the tail, it becomes evident that the use of a tail structure leads to lighter, more compact robots with high mobility on uneven terrain.

\section{HELIOS CARRIER}

The mobile platform introduced hereafter and equipped with the tail like mechanism, is part of a special robot team proposed for USAR operations. In particular it represents the first stage of a Japanese national founded project which aims to the development of a robotic system to be utilized in extreme environments such as rescue operations in heavily stricken urban areas. As presented in [7] the solution provided by the authors consists of five tracked platforms (two HELIOS IX vehicles and three simple carriers). These mobile bases are utilized to take sensors and measuring devices into heavily stricken areas for the realization of 3D maps and for collecting information on the explored areas. Because of weight and occupancy of the devices mounted on the vehicles, tilting motions must be addressed when moving over stairs and unstructured environments.

Figure 1 shows one of the three carriers equipped with the tail mechanism introduced earlier. The tail is composed of a cylinder, and it is made of a flexible material like rubber. By adjusting the flexibility of the material, one can determine how much impact can be absorbed by it, and how much the robot will bounce while climbing stairs. As presented in the test and experiments section, also with the simple fixed tail mechanism the tracked robot presents very good stability. However in order to utilize the mechanism also when descending the stairs and in case of sudden tilts, it is important to have a counter reaction. This can be obtained by supplying one dof (PITCH axis) to the mechanism.

As explained in the next section, using an attitude sensor installed inside the body and two distance sensors (connected to the front and to the back of the mobile base) it is possible to automatically trigger the tail actuation. Besides when the tail is not utilized, it can be set vertically with respect of the robot base in order to not obstruct pivot rotations in limited spaces. Eventually a protected camera (with $180^{\circ}$ view) can be installed at the tip of the linkage and aligned to the tail axis for driving the vehicle. For this reason a hollow pipe was utilized as tail in order to allow the passage of wires and the antenna.

\section{A. Mechanical design}

The base of the tail is connected to a worm gear, and rotates around two bearings connected to the base of the robot, as shown in figure 6 . The worm gear is driven by a worm screw, which is actuated by a motor installed on the base of the robot. Because of the worm gear, the tail cannot be back-driven, and is able to keep its position even when external forces are applied, with no additional consumption of power by the driving motor. By changing the angle of the tail, it can be lifted in order to make the body of the robot more compact, or to avoid obstacles. In addition, by controlling the angle, traversing obstacles becomes smoother and more controllable.

Figure 7 shows sequences of crawler robots climbing stairs. The robot on the left (a-b) has no tail, while the robot on the right $(\mathrm{c}-\mathrm{d})$ is equipped with the tail mechanism. 


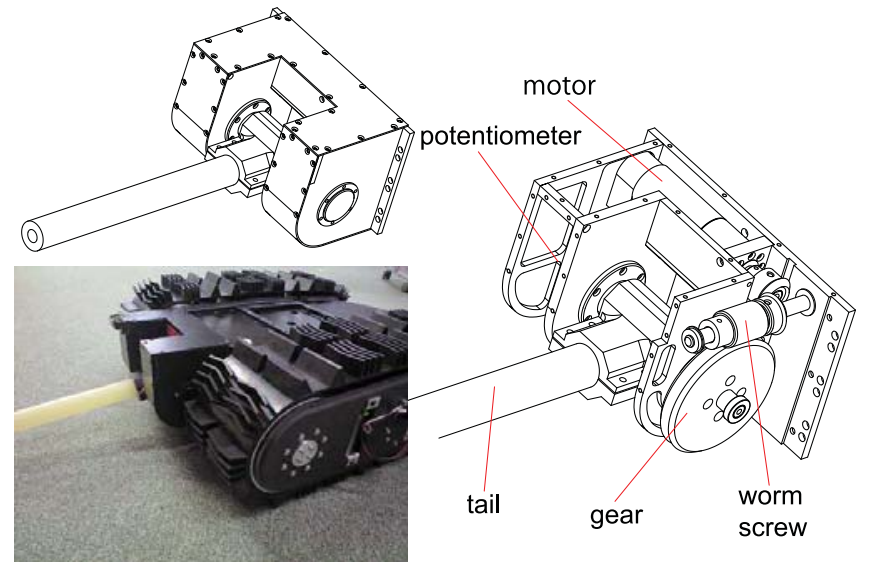

Fig. 6. Section view of the tail mechanism
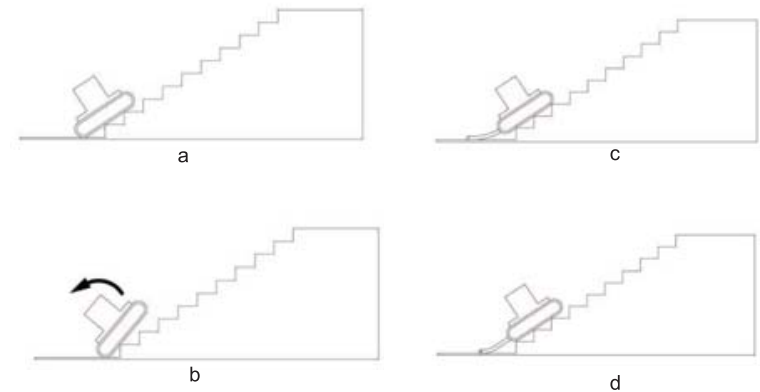

Fig. 7. Effect of the tail in ascending stairs
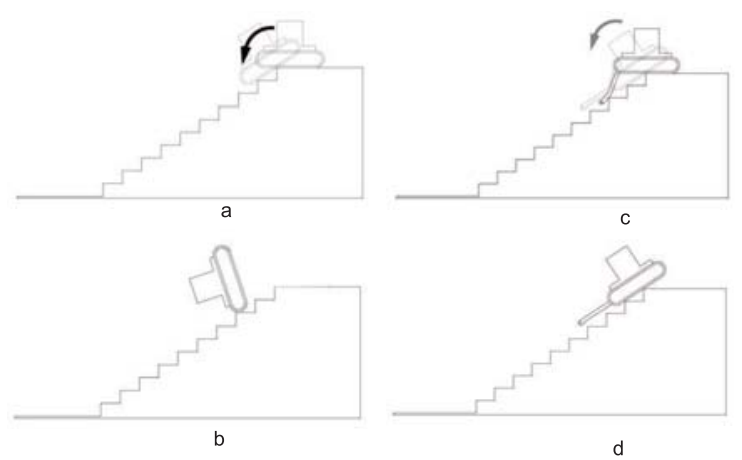

Fig. 8. Effect of the tail in descending stairs

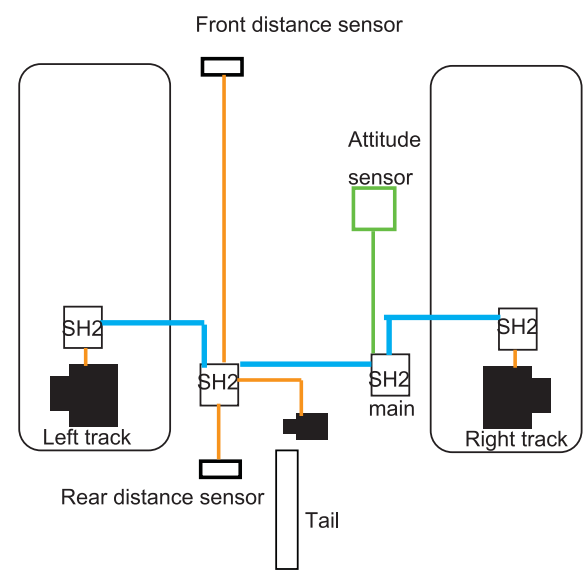

Fig. 9. Carrier robot architecture
Figure 8 shows the robot descending a flight of stairs. The robot without the tail falls suddenly once its center of mass is over the pivot edge, causing a loss of stability. The robot equipped with the proposed mechanism is able to change its pitch angle continuously with the help of the tail, and thus perform a controlled descent of the steps.

\section{B. Architecture and control method}

Figure 9 depicts the system architecture of the tracked platform. In the robot one main HiBot SH2 Tiny microcontroller is utilized for the reading of the attitude sensors data and for the communication to the external computer. Another SH2 Tiny is in charge for the control of the motor of the tail mechanism.

This microcontroller is also constantly acquiring the distance from the ground for both the front and rear side of the mobile platform with respect of the motion direction. According to the data received by CAN bus of the robot posture, this microcontroller enables and controls the actuation of the tail.

The tail is always actuated in position control and its target angle position is defined according to the distance sensors and the vehicle attitude readings.

1) descending stairs: Figure 10-a illustrates the case of the vehicle descending stairs.
If $r d>T h$ (where $r d$ is the rear ground distance and $T h$ is the track height) the tail is set downward of an angle $\beta$ in order to contact or at least decrease the distance between the robot and the first stair step. While the robot moves backward the base starts to tilt, a sudden $\alpha$ tilting would increase the tail angle of the same attitude $\alpha$. When the robot is moving downward $r d \leq T h$ thus the portion $\beta$ is removed. Afterwards the tail configuration is set only by the pitch attitude motions.

2) ascending stairs: Figure 10-b presents the case of the vehicle ascending stairs:

When the robot starts to move over the first step the only data added to the posture of the tail is the pitch attitude (if higher than a certain $\alpha$ degree) of the main body. This doesn't obstruct the climbing motion as the flexibility of the robot tail allows the treads to climb over the first step. Afterwards the motion continues over the stairs. When the vehicle is approaching the top, $f d>T h$ (case shown in figure 10-b), this enables a further rotation of the tail posture of $\beta$ in order to obtain a smooth motion without tilting.

While moving along the stairs if sudden Pitch accelerations occur, the tail is actuated in order to counter react to them and to maintain the stability of the robot. The simple control method is illustrated in the flow-chart of Figure 11.

$\alpha$ and $\beta$ must be set according to the type of stairs. Due 


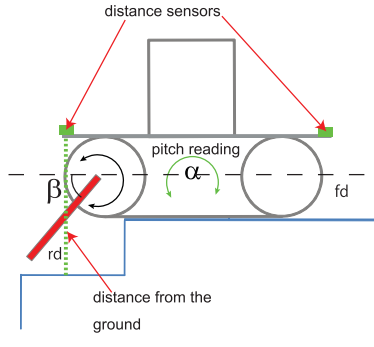

a

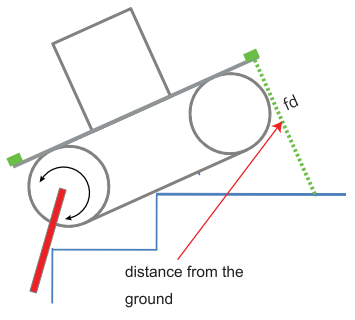

b
Fig. 10. Data reading for the tail control

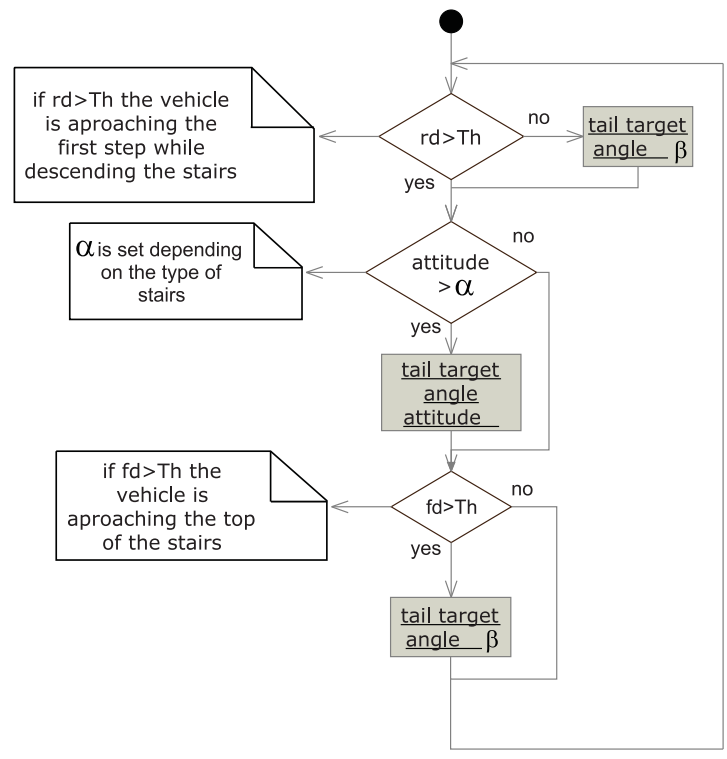

Fig. 11. Flow chart of the simple tail control method

to the flexibility of the tail, it is not necessary to set precise values for the above parameters, however to extend this control method, data acquired realtime from the environment should be included. In the experiments presented in the next section, $\alpha$ and $\beta$ were set in order to follow the inclination of the stairs profile at about $36^{\circ}$.

\section{TESTS}

Several tests have been carried out. Figure 12 shows the first validation test with using a non actuated tail system. The motion was successfully carried out however it was confirmed that when starting to descend, on the top and along the stairs it is necessary to actuated the tail for a stable and smooth motion.

The experiment presented in figure 13 was carried out with and without the proposed tail like mechanism. It is clear from the left side of the figure as a normal basic crawler vehicle is not capable to avoid tilting and to overcome simple obstacles.

In figure 14 the $\mathrm{CoG}$ of the robot is higher than in the previous experiments. Making use of the tail mechanism the carrier could ascend and descend the stairs without fails and/or tilting. Figure 14-1 shows the triggering made by

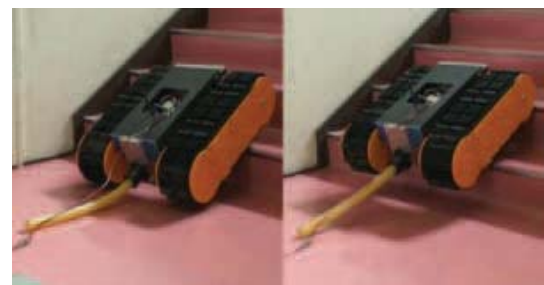

Fig. 12. First prototype with fixed tail
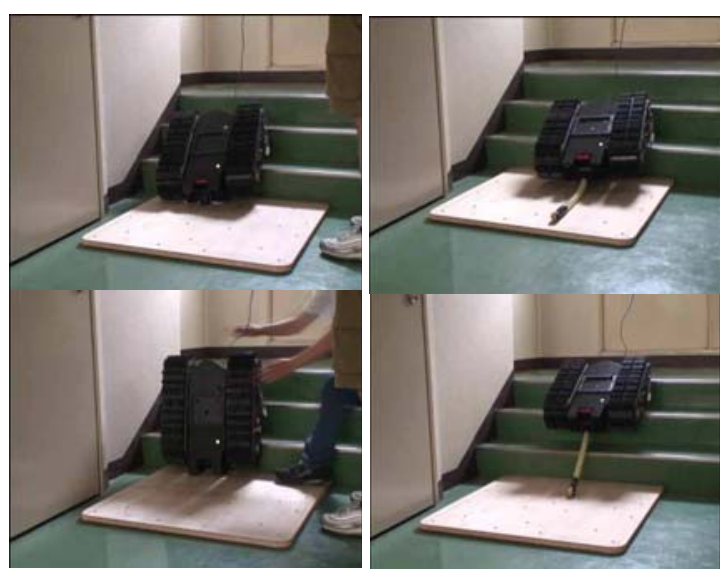

Fig. 13. Motion on stairs with and without tail

the distance sensor and the consecutive actuation of the tail toward the stair profile.

Due to the weight of the sensor device installed on the platform, (see frame number 3 and 4 ) the tail is widely bending. However, as this caused an increment of the vehicle inclination, by using the attitude sensor data, it was further rotated downward in order to compensate this issue. Another experiment is presented in figure 15; the tail bending is clearly visible when ascending and descending the stairs.

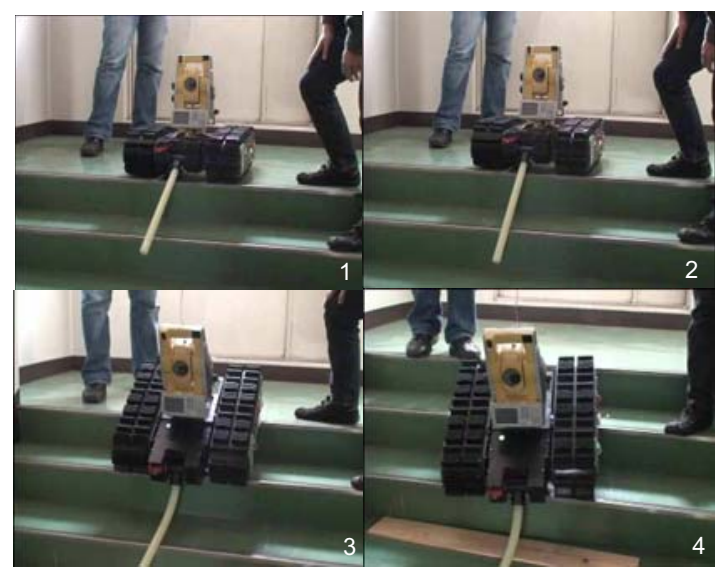

Fig. 14. Stair descending with high CoG

The mobile platform with the tail like mechanism was also tested during real experiments in one of the Tokyo subway stations. Figure 16 illustrates three frames of the motion in 


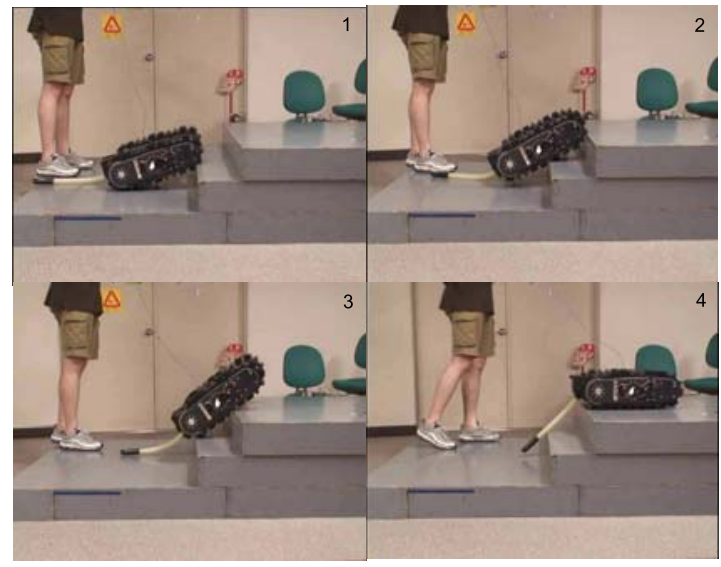

Fig. 15. Stair climbing

which one of the carriers was taken by the user from the first floor of the station down to the fourth floor of the train platform. As explained in section II, the middle frame shows clearly the carrier in its critical point; however because of the tail a good robot stability was successfully achieved. During this test the operator was concentrating only on the data reading on the environment supplying to the microcontroller only a simple command of moving forward or backward. The architecture presented earlier was in parallel taking care of the stability control along the stairs.

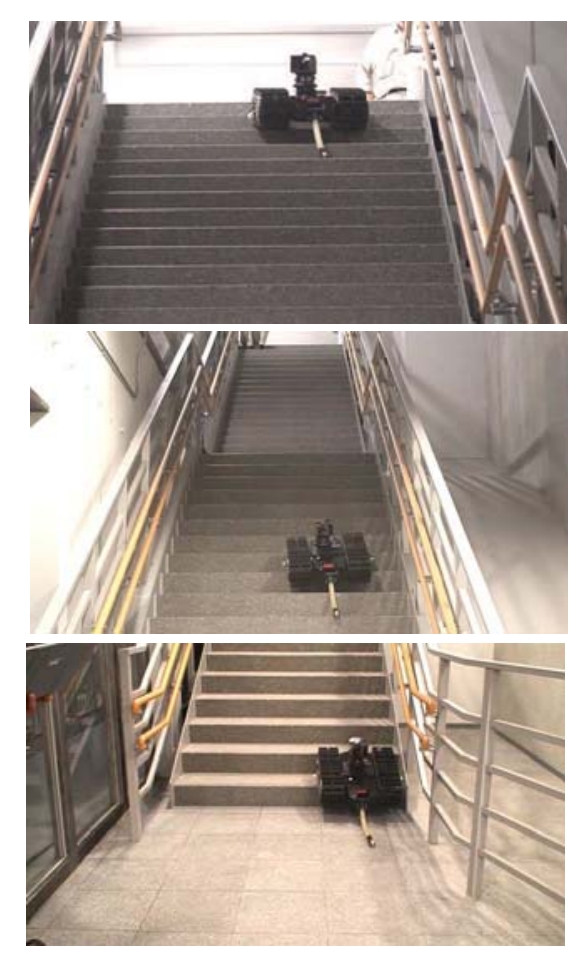

Fig. 16. Mobility experiment inside a Tokyo subway station

\section{CONCLUSION}

A new simple tail-like mechanism has been presented for the stability control of simple tracked mobile platforms. A basic model analysis has been carried out and the proposed solution has been applied to a team of tracked robots for search and rescue operations in urban areas. The effectiveness of the tail has been proved throughout several tests and experiments in simulated and real environments.

In future works, further analysis will be carried out in order to define a precise dynamic model of the system. Also a detailed study of the tail flexibility versus the specification of the mobile platform will be considered.

\section{ACKNOWLEDGMENTS}

The authors acknowledge the contribution of NEDO Japan who is sponsoring this research.

\section{REFERENCES}

[1] Y. Chiu,N. Shiroma,H. Igarashi,N. Sato,M. Inami and F. Matsuno, "FUMA: Environment Information Gathering Wheeled Rescue Robot with One-DOF Arm", Proceedings of the 2005 IEEE International Workshop on Safety, Security and Rescue Robotics, Kobe, Japan, 2005, pp 81-86.

[2] A. Birk,K. Pathak,S. Schwertfeger,W. Chonnaparamutt, "The IUB Rugbot: an intelligent, rugged mobile robot for search and rescue operations", International Workshop on Safety, Security, and Rescue Robotics (SSRR) IEEE press, 2006.

[3] P. Ben-Tzvi,A.A. Goldenberg and J.W. Zu, "Design, simulations and optimization of a tracked mobile robot manipulator with hybrid locomotion and manipulation capabilities",Proceedings of the 2008 IEEE/RSJ Conference on Intelligent Robots and Systems, pages 23072312, Pasadena, CA, USA, May 19-23, 2008

[4] J. Kyungmin et all, "A Remotely Operated Robotic System for Urban Search and Rescue", SICE-ICASE International Joint Conference 2006, Oct. 18-21, 2006 Bexco, Busan, Korea

[5] M.C. McHenry, K.Matthies, D.M.Hlmick, S.I.Roumeliotis, "Multisensor, high speed autonomous stari climbing", Proceedings of 2000 IEEE/RSJ International Conference on Intelligent Robots and Systems, pages 733-742, Lausanne, Switzerland, October 2002.

[6] M. Guarnieri and I. Takao and E. F. Fukushima and S. Hirose, " HELIOS VIII search and rescue robot: Design of an adaptive gripper and system improvements", Proceedings. 2007 IEEE/RSJ International Conference on Intelligent Robots and Systems, San Diego, USA, Nov 2007, pp. 1775-1780.

[7] M. Guarnieri and I. Takao and P. Debenest and K. Takita and E. F. Fukushima and S. Hirose, "HELIOS IX Tracked Vehicle for Urban Search and Rescue Operations: Mechanical Design and First Tests", Proceedings. 2008 IEEE/RSJ International Conference on Intelligent Robots and Systems, Nice, France, Sep 2008.

[8] Y. Hosoda, H. Yamamoto, M. Hattori, H. Sakiri, T. Iwamoto, M. Oowada, A. Kanno, Y.Saitou, "Swan: a robot for nuclear disaster prevention support", Journal of Advanced Robotics, volume 16, pp.485488, 2002

[9] J. Liu, Y. Wang, S. Ma, B. Li, "Analysis of stairs-climbing ability for a tracked reconfigurable modular robot", Proceedings of the 2005 IEEE International Workshop on Safety, Security and Rescue Robots, pages 36-41, Kobe, Japan, June 2005.

[10] C. Marques, J. Cristovao, P. Lima, J. Frazao, I. Riberio, R. Ventura, "Raposa: Semi-autonomous robot for rescue operations", Proceedings of the 2006 IEEE/RSJ Conference on Intelligent Robots and Systems, pages 3988-3993, Beijing, China, October 2006.

[11] L. Woosub, K. Sungchul, K. Munsang, S. Kyungchul, ” Rough Terrain Negotiable Mobile Platform with Passively Adaptive Double-Tracks and Its Application to Rescue Missions”, Proceedings. 2005 IEEE International Conference on Robotics and Automation, San Diego, USA, April 2005, pp. 1591-1596. 